\title{
A stochastic breakup model for Large Eddy Simulation of a turbulent two-phase reactive flow
}

\author{
W. P. Jones, A. J. Marquis, D. Noh* \\ Department of Mechanical Engineering, Imperial College London, Exhibition Road, London SW7 2AZ, UK
}

\begin{abstract}
The current work presents numerical investigations of model burner in which a non-swirling air-assisted methanol spray is injected using a pressure-swirl atomizer. A stochastic breakup model is formulated in the context of Large Eddy Simulation (LES) and validated by detailed comparisons of the results with measurements. An excellent agreement is achieved for the non-reactive case in terms of the dispersion of the spray, the mean droplet distributions and the time-averaged spray velocities. The transported probability density function $(p d f)$ equation/Eulerian stochastic field method are used to represent the interaction of turbulence and chemistry while the gas phase reaction of the methanol/air spray flame is described by a reduced reaction mechanism involving 18 chemical species and 84 elementary steps. The sub-grid scale ( $(g s)$ chemistry model in conjunction with the formulated breakup model are found to capture the influence of the flame on droplet dynamics together with the formation of a double reaction zone typically resulting from a polydisperse spray to a good accuracy.
\end{abstract}

Keywords:

Turbulent two-phase combustion, Large Eddy Simulation, Droplet breakup, PDF method, Pressure-swirl atomizer

\section{Introduction}

There are numerous engineering applications in industry such as diesel engines, gas turbines and rocket engines where liquid fuels are often delivered into combustion chambers via a means of atomization. When a column of liquid fuel is discharged into a gas, it becomes unstable and disintegrates into smaller droplets, forming a spray. The resulting spray then evaporates and creates fuel vapour that mixes with the surrounding gas and burns. This atomization process plays a crucial role in determining the global performance of two-phase combusting flows in terms of the rate of fuel consumption and pollutant emissions. In the present work, a probabilistic model for the atomization of sprays [1] is adopted in combination with an LES of the gas phase and further developed to simulate the methanol spray flame,

${ }^{*}$ Corresponding author

Email address: dongwon.noh10@imperial.ac.uk (D. Noh) 
studied experimentally by [2, 3]. The model for breakup consists of a stochastic formulation which reproduces the effects of the sub-grid scale ( $s g s)$ motions on droplet breakup with each breakup event being defined in a statistical manner through a Poisson release process. The evolution of droplet diameter through evaporation is also described in a probabilistic approach.

\section{Experimental configuration}

The spray flame configuration under investigation is that of McDonell et al. [2, 3]. A dilute methanol spray is injected using a Research Simplex Atomizer (RSA) manufactured by Parker Hannifin. A full description of the operating conditions can be found in [4]. The simplex atomizer has a flow number of 1.36 and a nominal spray angle of $85^{\circ}$. The injection assembly with a $4.9 \mathrm{~mm}$ diameter exit is mounted in a $495 \times 495 \mathrm{~mm}$ square duct. In the combustion chamber, there is an additional air passage co-flowing around the atomizer assembly at an average velocity of $1.0 \mathrm{~m} / \mathrm{s}$. All measurements were obtained at atmospheric pressures and room temperatures. The mass flow rate of air is $1.32 \mathrm{~g} / \mathrm{s}$ resulting in a pressure drop of $3.73 \mathrm{kPa}$ while the liquid fuel is injected at $1.26 \mathrm{~g} / \mathrm{s}$ corresponding to $\Delta p_{l}=420 \mathrm{kPa}$. This operating condition results in a Reynolds number of 20,000 based on the diameter of the injector assembly exit, $D$, and a bulk velocity of $62.5 \mathrm{~m} / \mathrm{s}$. A lifted flame arises, the base of which is located from 50 to $60 \mathrm{~mm}$ downstream of the injector. At Reynolds numbers greater than 22,640, the flame is found to blow off.

In the experimental work, gas phase velocities for both the single and two-phase flow were measured using phase Doppler interferometry (PDI). The PDI system was also utilised to obtain data regarding liquid volume flux, droplet velocity as a function of size classes and droplet size. Additionally, the fuel vapour concentration was measured using infrared extinction/scattering (IRES). All the quantities are provided at the following axial locations: 15, 25, 50, 75, 100 and $150 \mathrm{~mm}$ away from the exit of the nozzle.

\section{Numerical modelling}

\subsection{LES equations: Interaction of gas and liquid phase}

The filtered governing equations of mass, momentum and scalars (chemical species or enthalpy) for low-Mach number flows are as follows:

$$
\begin{gathered}
\frac{\partial \bar{\rho}_{g}}{\partial t}+\frac{\partial \bar{\rho}_{g} \tilde{u}_{i}}{\partial x_{i}}=\overline{\dot{S}}_{m} \\
\frac{\partial \bar{\rho}_{g} \tilde{u}_{i}}{\partial t}+\frac{\partial \bar{\rho}_{g} \tilde{u}_{i} \tilde{u}_{j}}{\partial x_{j}}=-\frac{\partial \bar{p}}{\partial x_{i}}+\frac{\partial \bar{\tau}_{i j}}{\partial x_{j}}-\frac{\partial \tilde{\tau}_{i j}^{s g s}}{\partial x_{j}} \\
+\bar{\rho}_{g} g_{i}+\overline{\dot{S}}_{m o m, i}
\end{gathered}
$$




$$
\begin{aligned}
\frac{\partial \bar{\rho}_{g} \tilde{\phi}_{\alpha}}{\partial t}+\frac{\partial \bar{\rho}_{g} \tilde{\phi}_{\alpha} \tilde{u}_{j}}{\partial x_{j}} & =\frac{\partial}{\partial x_{j}}\left(\frac{\mu}{\sigma} \frac{\partial \tilde{\phi}_{\alpha}}{\partial x_{j}}\right)-\frac{\partial \tilde{J}_{j, \alpha}^{s g s}}{\partial x_{j}} \\
& +\overline{\rho_{g} \dot{\omega}_{\alpha}(\underline{\phi}, T)}+\overline{\dot{S}}_{\alpha}
\end{aligned}
$$

where $\rho_{g}, \mu, u, p$ and $\phi_{\alpha}$ represent the fluid density, viscosity, velocity, static pressure and scalar quantities, respectively. The tensors, $\bar{\tau}_{i j}$ and $\tilde{\tau}_{i j}^{s g s}$, are the viscous and sub-grid scale stress tensors. The latter, defined as $\tilde{\tau}_{i j}^{s g s}=$ $\bar{\rho}_{g}\left(\widetilde{u_{i} u_{j}}-\tilde{u}_{i} \tilde{u}_{j}\right)$, is computed using the dynamically calibrated version of the Smagorinsky model [5].

The filtered conservation equations for specific mole number of the chemical species $[\mathrm{kmol} / \mathrm{kg}]$ contain the filtered net formation rates through chemical reaction, $\overline{\dot{\omega}_{\alpha}(\underline{\phi}, T)}$. The direct evaluation of these poses serious difficulties and to overcome these a joint $s g s$ probability density function $(p d f)$ approach is adopted to determine species composition and enthalpy. The modelled sgs-pdf equation is solved using the LES stochastic field method [6]. The coupling between the gaseous and liquid phases is incorporated by the addition of terms representing the source of mass, momentum and scalar quantities, $\overline{\dot{S}}_{m}, \overline{\dot{S}}_{m o m, i}$ and $\overline{\dot{S}}_{\alpha}$, respectively. The source terms are evaluated from $\overline{\dot{S}}=\frac{1}{\Delta^{3}} \sum_{p=1}^{N_{p}} \dot{S}^{(p)}$ where the summation is performed over the number of droplets present within a specific control volume, $\Delta$ is the filter width and $\dot{S}^{(p)}$ is the appropriate source term arising from the $p$-th droplet.

\subsection{PDF modelling of sprays}

The probabilistic representation of the dispersed phase [7] is adopted in the present work. The required filtered joint $p d f$ is $\bar{P}_{s p r}(\boldsymbol{V}, D, \Theta, N ; \boldsymbol{x}, t)$ in which $\boldsymbol{\Psi}=\{\boldsymbol{V}, D, \Theta, N\}$ is the corresponding phase space for the state vector, $\boldsymbol{\Phi}=\{\boldsymbol{v}, d, \theta, n\}$. The equation describing the temporal evolution of this joint $p d f$ can be written as:

$$
\frac{\partial \bar{P}_{s p r}}{\partial t}+\frac{\partial\left(\boldsymbol{a} \bar{P}_{s p r}\right)}{\partial \boldsymbol{V}}+\frac{\partial\left(\dot{\mathcal{D}} \bar{P}_{s p r}\right)}{\partial D}+\frac{\partial\left(\dot{\mathcal{T}} \bar{P}_{s p r}\right)}{\partial \Theta}+\frac{\partial\left(\dot{\mathcal{N}} \bar{P}_{s p r}\right)}{\partial N}=0
$$

where $\boldsymbol{a}, \dot{\mathcal{D}}, \dot{\mathcal{T}}$ and $\dot{\mathcal{N}}$ represent the conditional particle acceleration, the conditional rate of change of droplet diameter through evaporation, the conditional rate of change of droplet temperature caused by heat transfer from the surrounding gas and the conditional rate of change of the droplet number through droplet breakup process, respectively. These can be expressed in the general form:

$$
E\left(\frac{D \psi_{k}}{D t} \mid \boldsymbol{\Psi}=\boldsymbol{\Phi}\right) \quad \text { where } \boldsymbol{\Phi}=\boldsymbol{v}, d, \theta \text { and } n
$$

where $E\left(\frac{D \psi_{k}}{D t} \mid \boldsymbol{\Psi}=\boldsymbol{\Phi}\right)$ is the expectation of $\frac{D \psi_{k}}{D t}$ conditioned upon $\boldsymbol{\Psi}=\boldsymbol{\Phi}$ anywhere within the filter volume. These quantities are unknown and hence closure models are required.

In order to first model and solve Eq. (4), it is replaced with an equivalent system [8] of stochastic differential 
equations (sdes) which describe the trajectories of stochastic particles in the phase space, $\{\boldsymbol{V}, D, \Theta, N\}$. The unclosed terms appearing in Eq. (4) are decomposed into a deterministic part and a stochastic contribution. The deterministic contribution to the Lagrangian rates of change of relevant variables is determined using the filtered values of the gaseous phase properties at the particle location whereas the additional influence of unresolved fluctuations on the filtered Lagrangian rates of change requires a modelling procedure. In this work, a stochastic Markov model [9, 7] is adopted to characterise the effects of the sgs motions on the dispersion of droplets. The evaporation model of Abramzon and Sirignano [10] is used to calculate the deterministic rates of change of droplet temperature and mass while the Sherwood number is decomposed, like a classical decomposition of velocity components, in order to account for the influence of unknown sgs fluctuations on the evolution of droplet mass. Further details are given in [11]. In the following section, an improved version of the previously formulated breakup model [1] is described.

\subsection{Stochastic breakup model}

Droplet breakup processes are statistically treated through the conditional Lagrangian rate of change of the droplet number, $\dot{\mathcal{N}}$, appearing in Eq. (4). A Monte-Carlo trajectory integration is employed to reproduce the influence of the deterministic contribution and sgs fluctuations on the breakup of droplets. Each droplet fragmentation is described in a statistical manner using a discrete Poisson process while the size of daughter droplets is determined by a probabilistic model used to yield the $p d f$ of daughter droplet diameters depending on the properties of the turbulent gaseous flow. Following [12], the rate of change of the number density of droplets, $\dot{\mathcal{N}}$, due to fragmentation, can be written as:

$$
\begin{gathered}
\dot{\mathcal{N}}=\int_{D}^{\infty} m\left(D_{0}\right) f\left(D, D_{0}\right) \omega\left(\epsilon, D_{0}\right) n\left(D_{0}, t\right) d D_{0} \\
-\omega(\epsilon, D) n(D, t)
\end{gathered}
$$

where $m\left(D_{0}\right)$ is the mean number of droplets resulting from the fragmentation of a mother droplet of size $D_{0}, f\left(D, D_{0}\right)$ is the size distribution of daughter droplets produced upon the breakup of a mother droplet of size $D_{0}, \omega\left(\epsilon, D_{0}\right)$ is the breakup frequency of droplets of size $D_{0}$ and $n\left(D_{0}, t\right)$ is the mean number density of droplets of size $D_{0}$ at time $t$. Equation (6) represents the sum of the birth rate of droplets of size $D$ formed from the breakage of droplets larger than $D$ and the death rate of droplets of size $D$ due to their further disintegration into smaller ones. The evaluation of Eq. (6) necessitates the following models; the breakup frequency, $\omega(\epsilon, D)$, the $p d f$ of daughter droplet sizes, $f\left(D, D_{0}\right)$, and the expected number of daughter droplets, $m\left(D_{0}\right)$.

The current breakup model comprises a deterministic contribution mainly due to aerodynamic forces imposed on droplets by the surrounding gas and a stochastic formulation, which is a function of both the diameter of mother 
droplets, $D$, and the dissipation rate of turbulent kinetic energy, $\epsilon$, given by:

$$
\omega(D, \epsilon)=\frac{1}{\pi} \sqrt{\frac{8 \sigma}{\rho_{l}(D / 2)^{3}}}+K_{g} \frac{\sqrt{\beta(\epsilon D)^{2 / 3}-12 \sigma /(\rho D)}}{D}
$$

where $\omega$ is the total breakup frequency, $\sigma$ is the surface tension of liquid, $\rho_{l}$ is the liquid density and $\beta$ is an integration constant assigned a value of 8.2 [13]. The first term on the rhs of Eq. (7) is the deterministic component of the breakup frequency, which is an inverse function of the breakup time [14] for the Weber number $(W e)$ close to its critical value, i.e., $W e \approx 12$. Typical values of $W e$ in the experimental configuration under consideration are found to be in close proximity to the critical value. The second term, which contains the dissipation rate estimated from the LES solution via $\epsilon=2\left(v+v_{s g s}\right) \tilde{S}_{i j} \tilde{S}_{i j}$ where $\tilde{S}_{i j}$ is the filtered strain tensor, represents the $s g s$ contribution and is based on the work of Martínez-Bazán et al. [15] who studied experimentally the breakup of air bubbles in a stirred vessel. This model has also been applied to characterise the breakup of a liquid jet in a coaxial gas jet [16]. The empirical constant, $K_{g}$, which was originally set to 0.25 considering the breakup of air bubbles immersed in a water jet, is assigned a value of 0.10 consistent with measurements in the immediate vicinity of the nozzle.

The $p d f$ of daughter droplet sizes is based on a presumed binary breakup mechanism and, following [17], is given by:

$$
\begin{aligned}
& f^{*}\left(D^{*}\right)= \\
& \frac{D^{* 2}\left[D^{* 2 / 3}-\Lambda^{5 / 3}\right]\left[\left(1-D^{* 3}\right)^{2 / 9}-\Lambda^{5 / 3}\right]}{\int_{D_{\min }^{*}}^{D_{\max }} D^{* 2}\left[D^{* 2 / 3}-\Lambda^{5 / 3}\right]\left[\left(1-D^{* 3}\right)^{2 / 9}-\Lambda^{5 / 3}\right] d D^{*}}
\end{aligned}
$$

where $D^{*}=D_{1} / D_{0}$ and $\Lambda=D_{c} / D_{0}$. The critical diameter, $D_{c}=[12 \sigma /(\beta \rho)]^{3 / 5} \epsilon^{-2 / 5}$, simply represents the diameter of the largest droplet which will not undergo any fragmentation under the turbulent action of the flow. The number of droplets 'broken' is based on an expected droplet 'life' determined via a discrete Poisson process. The fragmentation model outlined above has previously been shown to yield good results for the breakup of droplets injected into a high speed air jet [1].

\subsection{Numerical investigation}

BOFFIN-LES, i.e., an in-house block-structured, parallel, variable density code with a low-Mach number approximation, is applied to the spray flame of interest. The reduced reaction mechanism of Li et al. [18] comprising 18 chemical species and 84 elementary steps is adopted to represent the gas phase reaction of the methanol/air flame while the unknown interaction of gas phase turbulence and chemistry at the sub-grid level is described by the stochastic fields/sgs-pdf equation approach; eight stochastic fields are used as found sufficient in the investigation of turbulent 
spray flames [11].

A fully structured, multi-block mesh of the burner, involving 1.4 million cells and 162 blocks, was constructed. The computational domain extending 30D radially and 100D axially is composed of the non-swirling atomizing air inlet, nozzle assembly in which the liquid fuel is injected, co-flow inlet, main combustion chamber and exit. The grid points across the atomizing air inlet are determined in a manner that the time-varying structure of turbulent inflow is well reproduced. A local refinement in the axial and radial grid spacings is made in regions near the nozzle exit where the breakup of methanol droplets, mixing of fuel vapour and air and shear layer of the expanding air jet take place. The size of the grid is stretched with a factor of below $10 \%$ along the axial and radial directions to ensure a fine resolution of computational grids in the vicinity of the nozzle exit. The size of the smallest cells is $0.1 \mathrm{~mm}$ with an aspect ratio of around unity while the maximum meshing space has a value of around $6 \mathrm{~mm}$ and is located close to the exit plane.

In order to represent a spatially and temporally inhomogeneous turbulent flow through the atomizing air inlet, a synthetic turbulence signal [19] is imposed on the velocity profiles at the inlet plane. Free slip conditions are applied at the outer surfaces of the domain and a convective outflow condition is applied at the exit plane. Based on the LISA model [20], droplets of diameter $81 \mu \mathrm{m}$ with a velocity of $22.8 \mathrm{~m} / \mathrm{s}$ are injected at random circumferential locations corresponding to the actual position of the injector whilst conserving the specified mass flow rate. In relation to the experimental findings of [21], the injection angle is allowed to have the highest probability around an estimated mean angle of $65^{\circ}$ and to vary between 40 and $90^{\circ}$ using a standard normal distribution. All the simulations are conducted with a time step of $0.4 \mu$ s ensuring a maximum CFL number of below 0.3 .

\section{Results and discussion}

In this section, a comparison of the numerical results with measurements is provided for the isothermal, nonreactive and burning spray cases at four axial locations $(25,50,100$ and $150 \mathrm{~mm})$.

\subsection{Isothermal flow without droplets}

Radial profiles of the mean and fluctuating axial gas velocities are shown in Figs. 1 and 2 As is evident, the simulated velocity profiles are in excellent agreement with those experimentally measured at all the axial locations; the magnitude of the jet velocity, the level of turbulence and the decay of the jet are well captured. Consequently, the specification of gas phase inlet/boundary conditions and the quality of the applied computational grid are validated. 


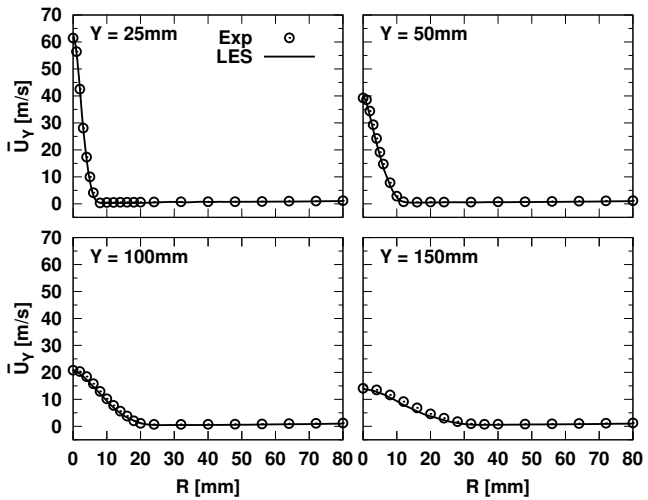

Figure 1: Radial profiles of the mean axial gas velocity for the isothermal case

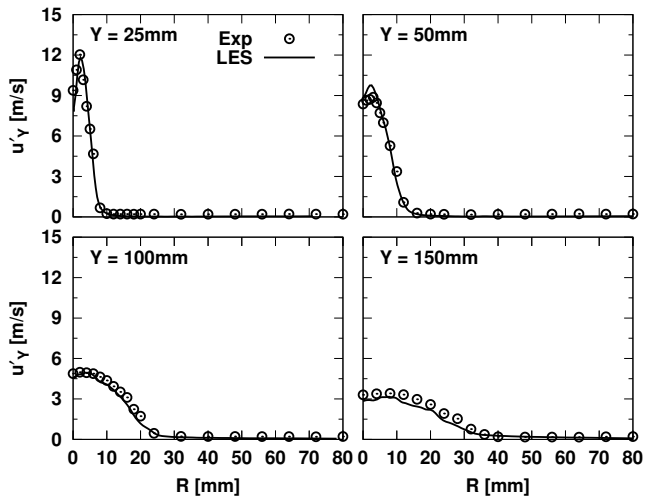

Figure 2: Radial profiles of the fluctuating axial gas velocity for the isothermal case

\subsection{The structure of the non-reactive spray and the spray flame}

Figure 3 represents a contour plot of the instantaneous gas velocity field together with a snapshot of individual droplets to provide a general impression on how the non-reactive spray is dispersed in the gas. A detailed image of droplet fragmentation near the atomizer is also shown. Initial droplets with a fixed diameter of about $81 \mu \mathrm{m}$ equal to the calculated liquid sheet thickness are injected at a range of the estimated full spray angles between 40 and $90^{\circ}$. The injected droplets then experience breakup events which create smaller droplets having a wide spectrum of diameters determined from Eq. (8). Generally, small droplets with relatively large fluctuating velocities are quickly convected towards the centreline due to the presence of an inwardly radial force imposed by the entrainment of co-flow into the jet. On the other hand, those with larger diameters are more likely to resist the momentum transfer from the surrounding gas and hence retain their initial trajectory for a longer distance. As a consequence, large drops are confined along the edge of the spray. A dense core of smaller droplets is observed to be surrounded by relatively large, slow drops travelling at radial locations away from the mixing layer of the jet. 


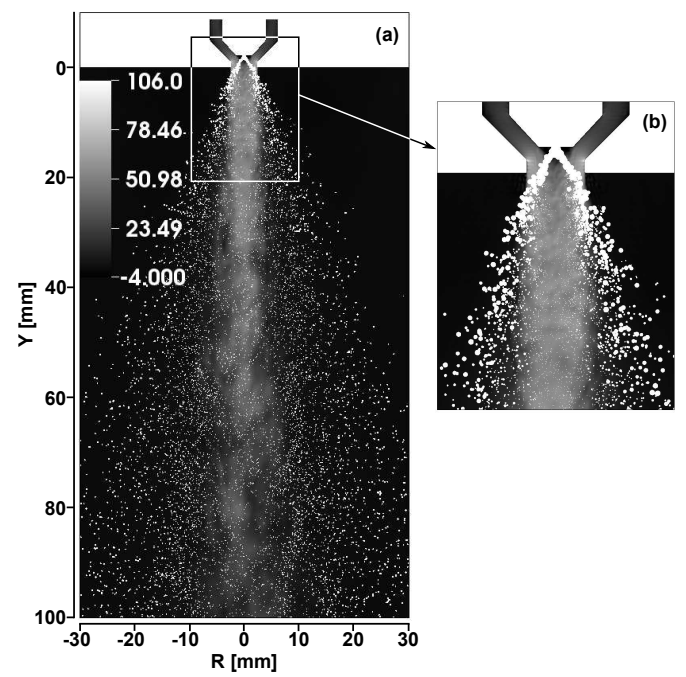

Figure 3: (a) The instantaneous gas phase velocity field with droplet motions and (b) a detailed view of breakup processes near the atomizer

Further information on the flame structure is provided by a contour plot of the time-dependent gas temperature field with the visible appearance of reacting drops as presented in Fig. 4 The overall flame structure reproduced in the flame calculation is in good agreement with that observed experimentally in the following aspects. In regions close to the atomizing assembly, the general properties of the reacting spray are comparable to those of the non-reactive spray; for example, the development of the central core highly loaded with small drops and the surrounding of the core by relatively large drops. This zone in advance of the onset of chemical reactions corresponds to a region where the breakup of hollow-cone liquid sheet primarily takes place with no apparent sign of combustion. In contrast to the structure of the non-reactive spray, the formation of reacting droplets shows distinct differences starting from an axial location, i.e., approximately between 50 and $60 \mathrm{~mm}$ [2]. In proximity to this axial location, a local void of large drops can be observed away from the centreline, which indicates the location of the reaction zone. The smallest drops along the centreline travel relatively further downstream, but evaporate fully at axial distances corresponding to a zone where the two flame fronts initially away from the centreline join together. Furthermore, it can be noticed that large droplets are also detected at radial locations beyond the location of the reaction zone.

The experimental findings of [22] are used to yield a further description of the simulated flame structure. Two snapshots of the instantaneous mass fraction of $\mathrm{OH}$ together with the corresponding droplet field and the stoichiometric mixture fraction are shown in Fig. 5 The methanol/air spray flame obtained in the LES simulation can be characterised by a double flame structure as a result of the polydisperse droplet distribution arising from the simplex nozzle. The double reaction flame involves two flame fronts diverging radially inwards or outwards on each half side of the combustion chamber. The flame fronts are initially joined together at the leading edge of the flame, which can 


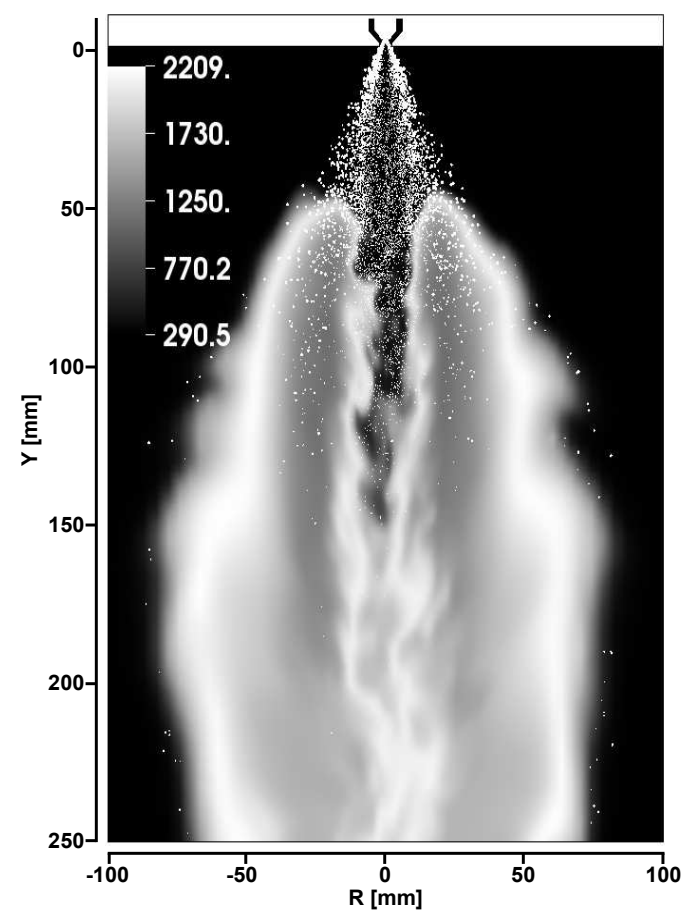

Figure 4: The instantaneous gas temperature with the visible appearance of the reacting spray

also be referred to as a stabilisation point. Depending on radial positions of these two flame structures relative to the axis of symmetry, they can be categorised into the inner and outer reaction zones.

The outer reaction zone burning in the co-flow with its low velocity exhibits less wrinkled and stable flame structures as it is well isolated from the turbulent nature of the jet. The appearance of the simulated $\mathrm{OH}$ mass fraction as a thin band along the stoichiometric mixture supports that the outer reaction zone may possess a typical characteristic of diffusion flames [23]. In contrast, the flame structure of the inner zone close to the stabilisation point is highly wrinkled and, to some extent, thinner owing to the interaction of turbulence with the flame front. This region burns like a diffusion flame and experiences relatively high strain rates when compared with the outer reaction zone [22]. Further downstream, the inner flame structure then experiences a transition to partially premixed combustion due to the role of turbulent mixing along the shear layer. This transition from diffusion-like to partially premixed flame is confirmed by the following observations that the widening of the simulated $\mathrm{OH}$ concentration is noticeable along the turbulent mixing layer and becomes more prevailing at around $\mathrm{Y}=150 \mathrm{~mm}$ where the penetration of the reaction zone to the centreline is complete. In premixed or partially premixed combustion, the broadening of the $\mathrm{OH}$ signal is obtained as a result of the presence of $\mathrm{OH}$ radicals within the hot combustion products [24].

The smallest droplets with high vaporisation rates travelling in the vicinity of the centreline produce the fuel vapour which is then supplied to sustain the inner reaction zone. On the other hand, relatively large drops are capable 
of penetrating the flame front along the inner reaction zone and then evaporate rapidly in the hot combustion products caused by the noticeable heat release from the oxidation process. This region serves as a fuel vapour source to maintain both the stable outer diffusion flame and the diffusive structure of the inner zone [24]. The flame is found to stabilise at roughly $\mathrm{Y}=50 \mathrm{~mm}$, which agrees well with the measured lift-off height.

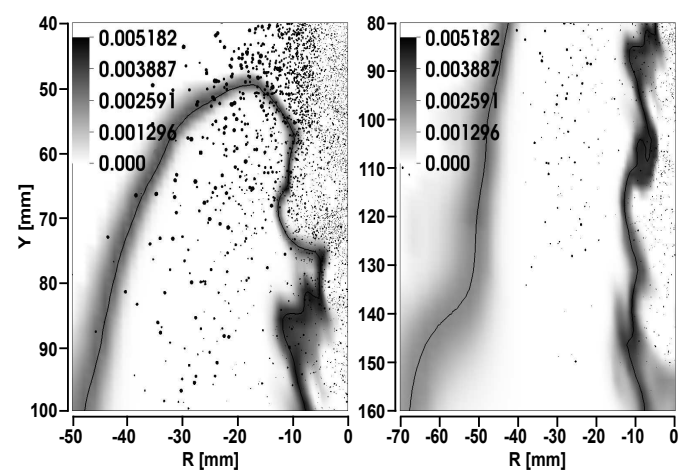

Figure 5: The instantaneous mass fraction of $\mathrm{OH}$ together with the corresponding droplet motions in the vicinity of the flame stabilisation point (left) and in the region where the reaction has penetrated to the centreline (right). Please note that the stoichiometric mixture fraction is highlighted in black solid lines

\subsection{Statistical results for the non-reactive and reacting spray cases}

The influence of the spray flame on the behaviour of droplets in terms of their size and velocity is investigated. Radial profiles of the arithmetic and Sauter mean diameters are shown in Figs. 6 and 7 The agreement between measured and simulated diameters is reasonably good for both the reacting and non-reacting cases although there are some discrepancies at the furthermost downstream location. This discrepancy is probably due to the simulated spreading rate of the non-reactive droplets being somewhat too low in this region. The radial profiles exhibit a typical characteristic of polydisperse sprays arising from hollow-cone simplex atomizers, i.e., minimum values along the centreline and maximum values towards the edge of the jet.

The simulated mean axial and radial velocities for all droplet size classes are compared to those measured in Figs. 8 and 9 The time-averaged axial spray velocities are well reproduced at all the axial locations. The simulated profiles of the mean radial component also agree well with experimental data up to the furthermost downstream measurement location where the magnitude is too low. This discrepancy in the radial velocity may be associated with the gas phase which is underestimated in comparison to that measured. In other regards, the general behaviour of the nonreactive spray is well captured. The influence of the reaction on both the axial and radial velocities is evident at axial locations starting from $\mathrm{Y}=100 \mathrm{~mm}$ with the increase in the magnitude of the droplet velocity being associated with the expansion of the gas phase during the reaction. 


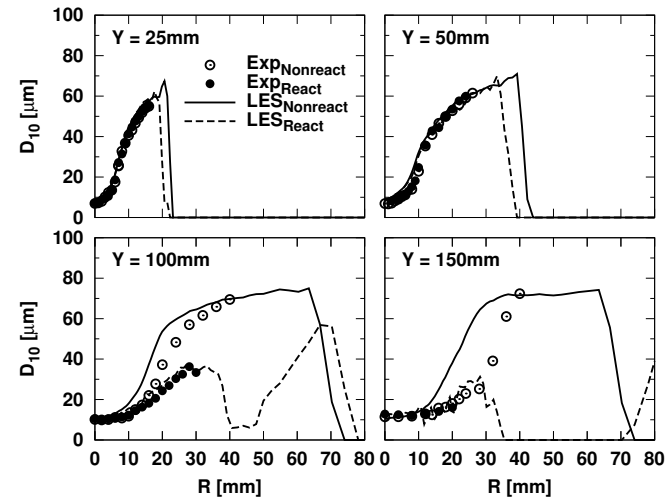

Figure 6: Radial profiles of the arithmetic mean diameter $\left(D_{10}\right)$ for the non-reactive and reacting cases

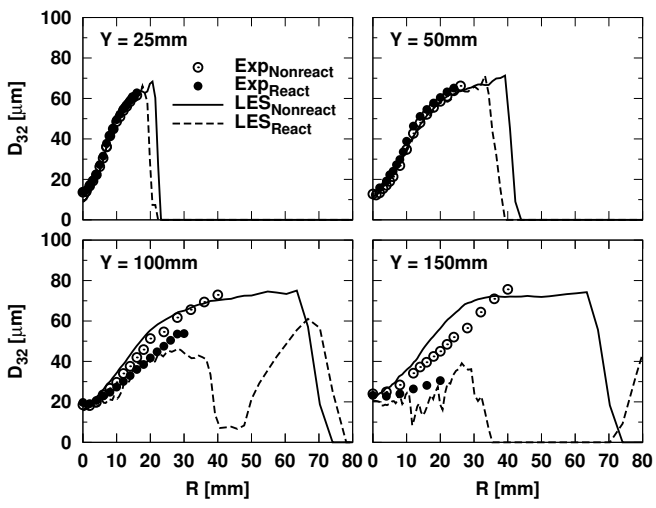

Figure 7: Radial profiles of the Sauter mean diameter $\left(D_{32}\right)$ for the non-reactive and reacting cases

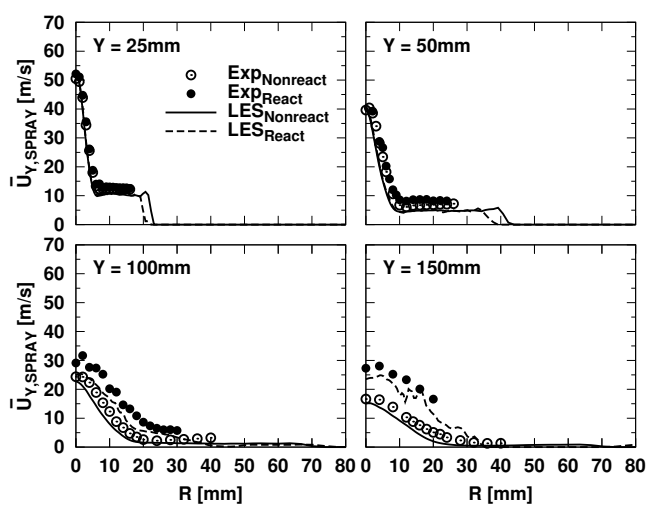

Figure 8: Radial profiles of the mean axial spray velocities for the non-reactive and reacting cases 


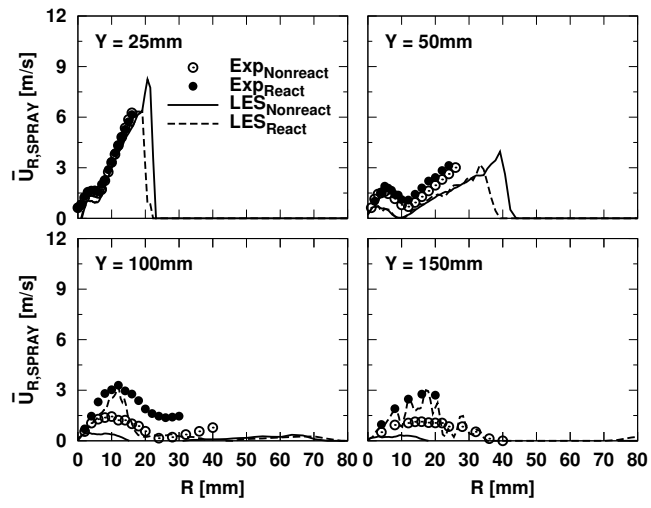

Figure 9: Radial profiles of the mean radial spray velocities for the non-reactive and reacting cases

\section{Conclusions}

A stochastic model for droplet atomization is presented and applied, in combination with LES and the $s g s-p d f$ transport equation/stochastic field method for gas phase combustion, to a methanol/air spray flame. Chemical reaction is described by a reduced mechanism involving 18 species and 84 reaction steps. For the methanol spray flame, the method yields results in overall good agreement with measurements for both the non-reacting and burning cases. The results for the non-reacting spray case are found to be, locally and globally, in excellent agreement with measurements in terms of the dispersion of the spray, the mean droplet diameters and the mean spray velocities. In the burning case, the measured lift-off height is also reproduced to a good accuracy. In addition, the comparison of the simulated statistics with measured profiles shows good agreement in terms of the influence of the flame on the droplet distributions. The acceleration of the spray arising as a consequence of the expansion of the gas phase during the reaction is also reasonably well captured.

\section{Acknowledgement}

The work was supported by the 7th Framework Programme of the European Union (FP7/2007-2013) under the Grant Agreement ACP0-GA-2011-265586 and used the ARCHER UK National Supercomputing Service http: //www . archer . ac .

\section{References}

[1] W. P. Jones, C. Lettieri, Phy. Fluids 22 (2010) 115106.

[2] V. McDonell, M. Adachi, G. S. Samuelsen, Atom. Spray 3 (1993) 389-410.

[3] V. McDonell, M. Adachi, G. S. Samuelsen, Atom. Spray 3 (1993) 411-436.

[4] V. G. McDonell, G. S. Samuelsen, J. Fluids Enging 117 (1995) 145-153.

[5] U. Piomelli, J. Liu, Physics of Fluids 7(4) (1995) 893-848. 
[6] W. P. Jones, S. Navarro-Martinez, Combust. Flame 150 (2007) 170-187.

[7] M. Bini, W. P. Jones, J. Fluid Mech. 614 (2008) 207-252.

[8] C. W. Gardiner, Handbook of Stochastic Methods for Physics, Chemistry, and the Natural Sciences, Springer, 2004.

[9] M. Bini, W. P. Jones, Phy. Fluids 19 (2007) 035104.

[10] B. Abramzon, W. A. Sirignano, Int. J Heat Mass Trans 32 (1989) 1605-1618.

[11] W. P. Jones, A. J. Marquis, D. Noh, Proc. Combust. Inst. 35 (2015) 1685-1691.

[12] J. C. Lasheras, C. Eastwood, C. Martínez-Bazán, J. Montanes, Int. J. Multiphase Flow 28 (2002) $247-278$.

[13] G. K. Batchelor, The theory of homogeneous turbulence, Cambridge university press, 1953.

[14] P. J. O'Rourke, A. A. Amsden, The TAB method for numerical calculation of spray droplet breakup, Technical Report, SAE Technical Paper, 1987.

[15] C. Martínez-Bazán, J. L. Montañés, J. C. Lasheras, J. Fluid Mech. 401 (1999) 157-182.

[16] J. C. Lasheras, E. J. Hopfinger, Annu. Rev. Fluid Mech. 32 (2000) 275-308.

[17] C. Martínez-Bazán, J. Rodríguez-Rodríguez, G. B. Deane, J. L. Montañés, J. C. Lasheras, J. Fluid Mech. 661 (2010) $159-177$.

[18] J. Li, Z. Zhao, A. Kazakov, M. Chaos, F. L. Dryer, J. J. Scire, Int. J. Chem. Kinet. 39 (2007) 109-136.

[19] M. Klein, A. Sadiki, J. Janicka, Journal of Computational Physics 186 (2003) 652-665.

[20] P. K. Senecal, D. P. Schmidt, I. Nouar, C. J. Rutland, R. D. Reitz, M. L. Corradini, Int. J. Multiphase Flow 25 (1999) $1073-1097$.

[21] T. Marchione, C. Allouis, A. Amoresano, F. Beretta, J. Propul Power 23 (2007) 1096-1101.

[22] S. K. Marley, E. J. Welle, K. M. Lyons, W. L. Roberts, Exp.1 Therm Fluid Sci. 29 (2004) 23-31.

[23] J. B. Kelman, A. R. Masri, Combust. Sci. Technol. 122 (1997) 1-32.

[24] M. A. Tanoff, M. D. Smooke, R. J. Osborne, T. M. Brown, R. W. Pit, in: Proc. Combust. Inst., volume 26, Elsevier, pp. 1121-1128. 